\title{
Toward whole-brain dopamine movies: a critical review of PET imaging of dopamine transmission in the striatum and cortex
}

\author{
Heather Liu ${ }^{1}$. Yasmin Zakiniaeiz ${ }^{2} \cdot$ Kelly P. Cosgrove ${ }^{2,3,4,5} \cdot$ Evan D. Morris ${ }^{1,2,3,5}$ \\ Published online: 25 October 2017 \\ (c) The Author(s) 2017, corrected publication December/2017. This article is an open access publication
}

\begin{abstract}
The mesocorticolimbic dopamine (DA) circuit, comprising the mesolimbic and mesocortical DA pathways, plays a crucial role in reward, cognitive control, and motivation. The positron emission tomography (PET) radiotracer, [C-11] raclopride, has been used widely to image DA receptors and DA changes in the mesolimbic pathway before and after pharmacological and behavioral challenges. In certain circumstances, properties of traditional kinetic models-used to analyze dynamic PET data—are not well-suited to describing the effects of stimulus-induced DA release. To combat model shortcomings, the authors have advanced a suite of models that characterizes PET data in the presence of timevarying DA release. We review select [C-11]raclopride studies of the striatum during cigarette smoking to illustrate the advantages of such models. DA receptors occur in lower density in the cortex than the striatum. This, as well as higher relative background signal, poses a serious challenge to quantitative PET of DA changes in the mesocortical system. Novel high affinity radioligands [F-18]fallypride and [C-11]FLB457 have been used to image mesocortical DA transmission. Models with time-varying terms may also hold the key to optimizing sensitivity to changes in mesocortical DA. As an illustration, we compare recent PET studies of the effect of stress on cortical DA release. Finally, we consider some challenges and strategies for further optimization of sensitivity of PET to stimulus-induced DA changes throughout the whole brain.
\end{abstract}

Keywords Dopamine release $\cdot$ Neuroimaging $\cdot$ Kinetic modeling $\cdot$ Model limitations $\cdot$ Smoking $\cdot$ Stress

\section{The mesocorticolimbic dopamine circuit}

Electronic supplementary material The online version of this article (https://doi.org/10.1007/s11682-017-9779-7) contains supplementary material, which is available to authorized users.

Evan D. Morris

evan.morris@yale.edu

1 Department of Biomedical Engineering, Yale University, New Haven, CT, USA

2 Interdepartmental Neuroscience Program, Yale University School of Medicine, New Haven, CT, USA

3 Department of Psychiatry, Yale University, New Haven, CT, USA

4 Department of Neuroscience, Yale University School of Medicine, New Haven, CT, USA

5 Department of Radiology and Biomedical Imaging, Yale University, Yale PET Center, P.O. Box 208048, New Haven, CT 06510, USA
Dopamine (DA) is an essential neurotransmitter for every-day brain functions including experiencing pleasure, regulating attention, and learning to control urges. There are two primary DA pathways in the brain that play fundamental roles in these functions - the mesolimbic and the mesocortical DA pathways. In the mesolimbic DA pathway, a rewarding stimulus, such as food or drug, stimulates DA neurons in the ventral tegmental area (VTA) leading to DA release in the ventral striatum (aka., nucleus accumbens). The mesocortical DA pathway also originates in the VTA, but produces DA release in the prefrontal cortex (PFC) (Swanson 1982). The mesolimbic pathway is critical for reinforcing motivated behavior (e.g., drug use), whereas the mesocortical pathway underlies emotional response and cognitive control, such as learning to inhibit drug use (Salamone and Correa 2002; Berridge 2007; Robinson and Berridge 1993; Schultz 2007; Dichter, Damiano, and Allen 2012). 
Dysfunction in one or both corticostriatal DA circuits has been thought to underlie psychiatric diseases such as addiction, attention-deficit hyperactivity disorder, and schizophrenia (Dichter et al. 2012). However, the precise mechanisms of dysregulation in these disorders are currently unknown. Histochemical, lesion and microdialysis studies in rodent brains were first used to identify the DA pathways (Kebabian et al. 1972; Willuhn et al. 2010). Unfortunately, these experimental tools (1) are limited by low spatial and temporal resolution, (2) cannot be used to record DA transmission in multiple brain regions simultaneously and, (3) are unsuited for clinical populations. With advanced techniques for analyzing PET data, we may be able to measure both the spatial distribution and the time course of DA transmission in humans in vivo and relate the observed patterns to behavioral performance and/or clinical outcomes.

A story is emerging that temporal patterns of DA transmission in response to a stimulus encode important information that may be relevant for understanding drug addiction, and treatment. These patterns may differ by location. Volkow and Swanson linked temporal patterns of [C-11]-cocaine uptake-an indirect marker of elevated synaptic DA - to temporal patterns of subjective reports of "feeling high" and "drug craving" for cocaine (Volkow and Swanson 2003). Rapid elevation of DA has thus come to be associated with fast onset of drug high and drug craving. Based on microdialysis work in rats, it has been hypothesized that the partial nicotinic agonist, varenicline ("Chantix") reduces the reinforcing properties of nicotine by eliminating sharp peaks in the DA response to nicotine (Rollema et al. 2007). Microdialysis and [C-11]FLB457 PET have been used together to examine DA transmission in the cortex of monkeys, leading to claims that DA transmission may be slow ("therapeutic") in the cortex and fast ("addictive") in the striatum (Jedema et al. 2014). These distinctions would be consistent with postulated functions of the mesolimbic and mesocortical systems, respectively.

Here, we review select PET papers reporting the effects of smoking or behavioral tasks on DA transmission. The papers help to illustrate the need for sophisticated modeling to properly detect and quantify the contribution of the DA signal to the PET data. These studies and further methodological refinements can lay the foundation for detailed characterization of both the mesolimbic and mesocortical DA loops. Better spatiotemporal characterization of DA transmission will provide a more complete picture of the workings of the dopaminergic circuitry. Our first illustration is based on work using [C-11] raclopride to image mesolimbic DA activity. The second illustration contrasts two reports of imaging behavior-induced DA transmission in mesocortical areas.

\section{[C-11]raclopride has been used extensively to image the striatum}

[C-11]raclopride (RAC) is a DA D2/D3 antagonist that is used for imaging dopamine (DA) receptors in the striatum. RAC is well-characterized and widely-used to study changes in endogenous striatal DA levels. It has been shown to be well-suited, kinetically, to displacement by DA changes caused by amphetamine (Morris and Yoder 2007). In the 20 years since the groundbreaking study by Koepp and colleagues demonstrating DA release during a goal-oriented motor task (Koepp et al. 1998), there have been about 150 papers using RAC and PET to study drugor behavior-induced DA changes (PubMed search terms: 'dopamine release', 'raclopride', 'PET', 'human'). For a thorough review, with particular focus on DA response to behavioral and cognitive challenges, see Egerton et al. (2009).

The Egerton review also covers PET imaging of drug challenges, including nicotine. Our focus has been on smoking. Nicotine binds to nicotinic acetylcholine receptors (nAChRs) on DA neurons in the VTA to activate DA pathways which manage reward and reinforcement (Imperato and Mitchell 1986). Suppl. Table 1 lists studies from the last 15 years that investigated nicotine-induced DA release with RAC. Binding potential relative to nondisplaceable tissue uptake $\left(\mathrm{BP}_{\mathrm{ND}}\right)$ (Innis et al. 2007), a measure of receptor availability, was used to estimate DA receptor level during each scan. The change in $\mathrm{BP}_{\mathrm{ND}}\left(\Delta \mathrm{BP}_{\mathrm{ND}}\right)$ was used as a measure of DA release during smoking. In general, the studies found that $\mathrm{BP}_{\mathrm{ND}}$ decreased due to the smoking stimulus. However, the magnitude of DA release was highly inconsistent, both across investigators and within the same group of investigators. The three studies (Table 1) performed by Brody et al. (Brody et al. 2004, 2006; Brody et al. 2010) can serve as an instructive case study for understanding the challenges of measuring stimulus-induced DA release with PET.

In each instance, investigators used an Equilibrium analysis (Watabe et al. 2000) to estimate $\mathrm{BP}_{\mathrm{ND}}$ before and after the stimulus. The stimulus (smoking) was performed at 50 min during a break in scanning although the infusion of tracer continued throughout. In the first study, the investigators recorded a $26-37 \%$ drop in $\mathrm{BP}_{\mathrm{ND}}$ (increase in DA) from before to after the smoking break in those who smoked. They employed apparently identical scanning and analysis procedures in the two follow-up studies. Curiously, they were unable to reproduce the same large change in $\mathrm{BP}_{\mathrm{ND}}$ as measured in the first study; the latter studies found only 8-9\% change in $\mathrm{BP}_{\mathrm{ND}}$. What could be at the root of such an inconsistency? 
Table 1 Comparison of smoking studies using RAC performed by Brody et al.

\begin{tabular}{|c|c|c|c|c|}
\hline Author/Journal & Cohort & Smoking stimulus & Data window & Findings \\
\hline Brody et al. (2004), Am J Psychiatry & $\begin{array}{l}20 \text { ND: } 10 \\
\text { smoked, } \\
10 \\
\text { abstained }\end{array}$ & $\begin{array}{l}1 \text { cig. outside scanner } 50 \text { min post- } \\
\text { injection }\end{array}$ & $10 \mathrm{~min}$ & $\begin{array}{l}26-37 \% \text { reduction in } \mathrm{BP}_{\mathrm{ND}} \text { in left } \mathrm{VC} \text {, } \\
\mathrm{NACC} \text {, and left VP }\end{array}$ \\
\hline $\begin{array}{l}\text { Brody et al. (2006), Arch Gen } \\
\text { Psychiatry }\end{array}$ & $\begin{array}{l}45 \text { ND: } 25 \\
\text { smoked, } \\
10 \\
\text { abstained }\end{array}$ & $\begin{array}{l}1 \text { cig. inside scanner } 50 \text { min post- } \\
\text { injection }\end{array}$ & $30 \mathrm{~min}$ & $\begin{array}{l}8.4 \pm 13.8 \% \text { reduction in } \mathrm{BP}_{\mathrm{ND}} \mathrm{VC} \text { and } \\
\quad \mathrm{NACC}\end{array}$ \\
\hline $\begin{array}{l}\text { Brody et al. (2010), Psychiatry Res. } \\
\text { Neuroimaging }\end{array}$ & $43 \mathrm{ND}$ & $\begin{array}{l}1 \text { cig. outside scanner } 50 \text { min post- } \\
\text { injection }\end{array}$ & $30 \mathrm{~min}$ & $\begin{array}{l}8.6 \pm 1.6 \% \text { reduction in } \mathrm{BP}_{\mathrm{ND}} \text { in } \mathrm{VC} \\
\text { and } \mathrm{NACC}\end{array}$ \\
\hline
\end{tabular}

$B P_{N D}$ binding potential, $N D$ nicotine dependent, cig cigarette, $V C$ ventral caudate, $N A C C$ nucleus accumbens

\section{Time-invariant models fail to accurately describe smoking-induced DA release}

We have previously shown that inconsistency in the results of the above-mentioned smoking studies could be attributed to limitations of conventional models, such as SRTM, Logan plot, or Equilibrium analysis (Sullivan et al. 2013; Yoder et al. 2004). The calculated endpoint, $\mathrm{BP}_{\mathrm{ND}}$, stipulates a constant level of DA binding throughout the duration of the scan. A consequence of applying the conventional models, which rely on time-invariant parameters, is that $\mathrm{BP}_{\mathrm{ND}}$ becomes sensitive to the amount of data used poststimulus. To demonstrate, Sullivan et al. simulated time activity curves (TACs) that incorporated a brief deflection indicative of transient DA elevation post-stimulus. Figure 1 demonstrates that inclusion of data during the transient DA elevation lowers the fitted curves relative to the baseline trajectory, producing substantial $\Delta \mathrm{BP}$ - at least for narrow data windows. As the data window is widened to include recovery after transient DA release, the fitted curves are less responsive to the deflection and the estimated $\Delta \mathrm{BP}$ is attenuated. Since the two follow-up studies performed by Brody et al. $(2006,2010)$ used a wider data window than the first study ( $30 \mathrm{vs.} 10 \mathrm{~min}$ ), it follows that the estimated $\Delta \mathrm{BP}$ of the latter studies would be smaller.

\section{The linear parametric neurotransmitter PET model}

The conflicting results catalogued in Table 1 (and Suppl. Table 1) indicate that $\triangle \mathrm{BP}_{\mathrm{ND}}$ may be an inconsistent estimator of smoking-induced DA release. Traditional models that estimate $\mathrm{BP}_{\mathrm{ND}}$ do not contain explicit functions to describe short-lived neurotransmitter responses. By default, they assume that DA is constant-whether at baseline, or at some other value. Consequently, these models are unable to reliably capture transient responses (Yoder et al. 2004).
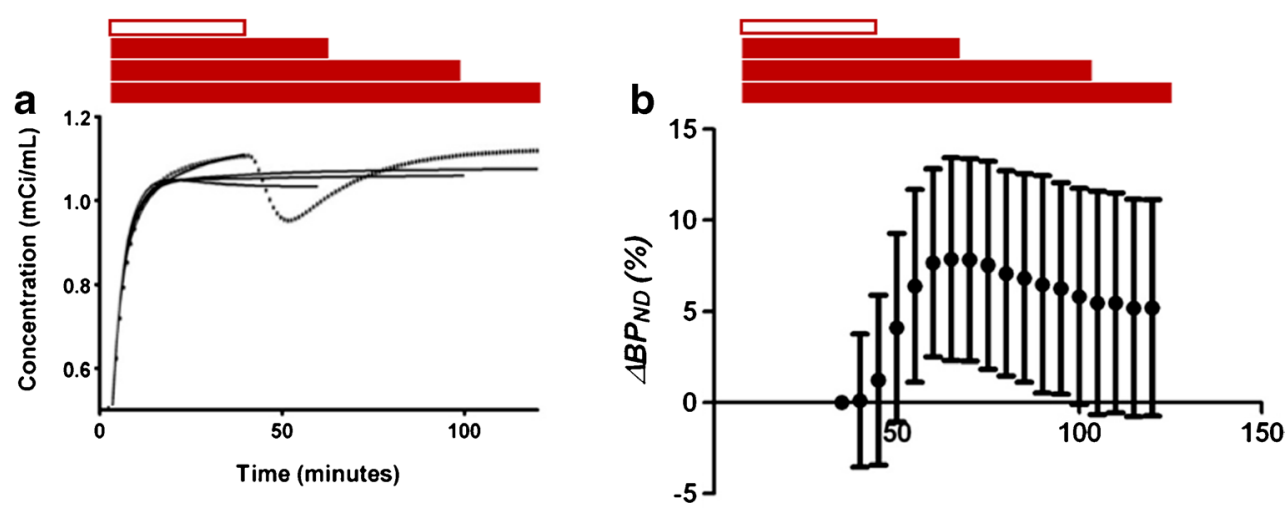

Fig. 1 Simulated PET TACs including the effect of transient DA release. Hollow bars: data window for baseline fit, solid bars: data windows for fits including DA transient. (a) effect of data window on fitted curves with SRTM (solid: fitted curves, dotted: simulated original TAC). Note solid curve that fits the data perfectly prior to

the effect of the transient at $40 \mathrm{~min}$. (b) effect of data window on estimated change in $\mathrm{BP}_{\mathrm{ND}}$ with SRTM, based on 100 simulated noisy TACs. Modified from Sullivan et al. (2013), Am J Nuc Med Mol Imaging 
The deficiencies in traditional models demand more flexible methods to fully characterize DA transmission. To resolve this deficiency, we have developed the linear parametric neurotransmitter PET (lp-ntPET) model (Kim et al. 2014; Morris et al. 2008, 2005; Normandin et al. 2012; Wang et al. 2016; Normandin and Morris 2006). Lp-ntPET is an extension of MRTM (Ichise et al. 2003).

$$
C_{T}=R_{1} C_{R}(t)+k_{2} \int_{0}^{t} C_{R}(u) d u-k_{2 a} \int_{0}^{t} C_{T}(u) d u-\gamma \int_{\mathbf{0}}^{\mathbf{t}} \mathbf{C}_{\mathbf{T}}(\mathbf{u}) \mathbf{h}_{\mathbf{i}}(\mathbf{u}) \mathbf{d u}
$$

Lp-ntPET (Eq. 1) is the union of two models. The model is comprised of the left-hand term (conventional MRTM for tracer concentration, $\mathrm{C}_{\mathrm{T}}(\mathrm{t})$ ), and the righthand term in bold font (effect of time-varying DA, $h_{i}(t)$ ). In practice, the optimal $h_{i}(t)$ is selected from a library of possible basis functions, which are typically configured as gamma variates but could take any form. Data from microdialysis studies support the use gamma variate functions for describing neurotransmitter release in response to stimuli Narendran et al. (2014; Tanda et al. 2015; Jedema et al. 2014). The gamma-variate functions used in lp-ntPET are each characterized by a distinct 'sharpness $(\alpha)$ ', 'response start time $\left(\mathrm{t}_{\mathrm{D}}\right)$ ', 'peak response time $\left(t_{\mathrm{P}}\right)$ ', and 'peak height $(\gamma)$ '. Figure 2 shows a representative (partial) set of bases that describe possible DA responses. Any of the conventional models would simply describe the DA response as a constant value for the entire scan.

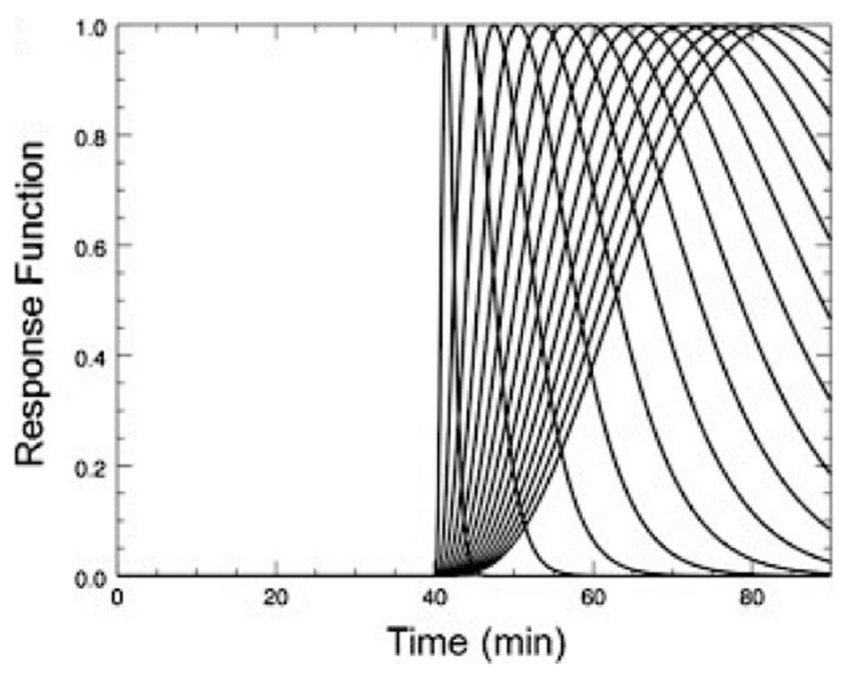

Fig. 2 A partial set of gamma-variate shaped DA response functions as described by lp-ntPET. Modified from Kim et al. (2014), Human Brain Mapping
DA release is deemed to be significant-and retainedonly in those cases (voxels) for which inclusion of the final term in Eq. 1 containing the time-varying response function yields a better fit than the time-invariant model. Significance is determined by the F-test comparing the sum of squares from lp-ntPET to the sum of squares from MRTM (left hand term in Eq. 1 only). The F-statistic accounts for differences in degrees of freedom of the two models. That is, it penalizes the fit of lp-ntPET for additional model parameters.

Applied at the voxel level, the primary endpoint of lpntPET becomes a subject-specific spatiotemporal pattern of (significant) DA release over the course of the scan-which can be thought of as a 'dopamine movie'. The action in a dopamine movie derived from RAC scans is confined to the striatum. Dynamic DA release can be detected with a temporal resolution on the order of minutes (Cosgrove et al. 2014; Normandin et al. 2012).

\section{Lp-ntPET is sensitive to subtle spatiotemporal fluctuations in DA during smoking}

We have applied lp-ntPET to study the brain's response to smoking Cosgrove et al. (2014). Eight male and eight female smokers were matched for smoking history and habits. The groups did not differ in radioactivity dose, injected mass of cold tracer, or craving prior to the scan. Overnightabstinent subjects smoked inside the scanner, starting 35 minutes ${ }^{1}$ following the initiation of a bolus plus infusion of RAC. Correction for head motion was applied at the eventlevel during reconstruction (Carson et al. 2004). Lp-ntPET was applied in all voxels in the pre-commissural striatum (Kim et al. 2014).

Significant sex differences were found in both the spatial extent and timing of activation during smoking (Fig. 3). Men activated consistently and robustly in the right ventral striatum while women did not. This finding is consistent with other research indicating that men smoke more for rewarding and reinforcing properties of nicotine compared to women (Perkins et al. 1996, 2002).

A consequence of lp-ntPET applied voxelwise is that the DA response can be visualized as a series of DA images over time. Online Resource 1 is a 'movie' of the DA response to smoking. Smoking began at $45 \mathrm{~min}$ for the subject; the response was estimated only in the pre-commissural striatum.

\footnotetext{
${ }^{1}$ Our protocol originally initiated smoking at $45 \mathrm{~min}$. It was modified to $35 \mathrm{~min}$, after we completed an optimization simulation study (Wang et al. 2016) that showed that sensitivity to DA is increased by an earlier stimulus. The optimal smoking time finds a balance between (1) acquiring sufficient baseline data before smoking to characterize tracer parameters, and (2) minimizing the effects of increased measurement noise in late-time data.
} 
Fig. 3 "Probability of Activation" maps for male (M) and female (F) smokers. Note difference between sexes in right ventral striatum (rVS). A permutation test performed using all 16 subjects showed mean difference in number of activated voxels in rVS between $\mathrm{M}$ and $\mathrm{F}$ was highly significant $(p<0.01)$. Right brain is on right. Modified from Cosgrove et al. (2014), J Neurosci

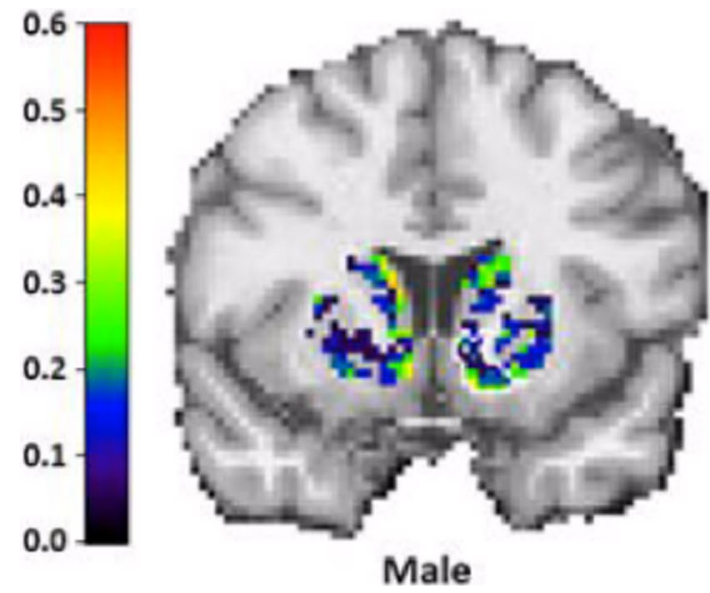

The emergence of a significant finding of sex differences from a small cohort suggests that lp-ntPET is more sensitive to short-lived fluctuations in DA than traditional models. Smoking studies prior to Cosgrove et al. 2014 (Suppl. Table 1) could not provide any temporal information about the DA response. It appears that lpntPET can extract more nuanced information about DA release than was possible previously.

\section{High affinity tracers have been used to image cortical regions}

The binding affinity of RAC $\left(\mathrm{K}_{\mathrm{D}} \sim 1-10 \mathrm{nM}\right)$ (Farde et al. 1986; Hall et al. 1988) is adequate for imaging D2/D3 receptors in areas with high DA receptor density relative to nonspecific uptake. However, RAC's low signal-to-background ratio and low DA receptor density, extrastriatally, leads to unreliable quantification and limits use of RAC to the striatum (Egerton et al. 2009). Thus, RAC imaging can provide only a partial picture of the full corticolimbic circuitry.

Development of high-affinity radioligands, [F-18]fallypride and [C-11]FLB457, has made it possible to image DA receptors in areas of low DA receptor density such as the cortex (Mukherjee et al. 1999; Olsson et al. 1999). Human imaging studies within the past decade using [F-18]fallypride and [C-11] FLB457 have begun to elucidate cortical function during various DA-releasing pharmacological and behavioral challenges. A non-exhaustive list of these studies is given in Suppl. Table 2.

Some studies (Suppl. Table 2) used the Montreal Imaging Stress Task (MIST) to assess changes in DA in response to a behavioral stressor. MIST (Dedovic et al. 2005) is a well-validated model of psychosocial stress that requires the participant to complete mental arithmetic problems that are manipulated to prevent subjects from achieving their expected performance. This perceived underachievement is supplemented with "evaluative threats" via negative feedback and monitoring by investigators. Evaluative threats have been shown to significantly raise cortisol levels in participants (Dedovic et al. 2005).

\section{Studies using [F-18]fallypride and MIST provide a direct comparison of kinetic models}

Table 2 provides details of two particular studies from Suppl. Table 2: Lataster et al. (2011) and Nagano-Saito et al. (2013). These studies together constitute an interesting
Table 2 Comparison of Lataster (2011) and Nagano-Saito (2013) by cohort demographics, experimental design, and analysis method

\begin{tabular}{lll}
\hline & Nagano & Lataster \\
\hline Tracer & {$[$ F-18]fallypride } & [F-18]fallypride \\
$\mathrm{N}$ & $11 \mathrm{HC}: 11 \mathrm{M}$ & $12 \mathrm{HC}: 8 \mathrm{M}, 4 \mathrm{~F}$ \\
Age & $21.5 \pm 3.3$ & $38.8 \pm 15.8$ \\
Stimulus & MIST & MIST \\
Smoothing (FWHM) & $8 \mathrm{~mm}$ & $4 \mathrm{~mm}$ \\
Mask location & Frontal gray matter & Prefrontal regions (BA9; BA10; BA11; \\
& & BA24; BA32; BA44; BA45; BA46; BA47) \\
Search volume & $207 \mathrm{~mL}$ & $231 \mathrm{~mL}$ \\
MC correction method & Gaussian random field & Simes-Hochburg (FDR), $p=0.05$ \\
& (FWER), $p=0.05$ & \\
Model/endpoint & SRTM, BP & LSD \\
\hline
\end{tabular}


comparison of methods. Both used [F-18]fallypride to image extrastriatal DA changes in healthy control subjects while performing MIST. Because the studies used the same tracer, stimulus, and a similar number of subjects, it would be reasonable to expect comparable results. The two research teams, however, applied different kinetic models with different calculated endpoints to their data: Nagano-Saito used SRTM (Lammertsma and Hume 1996) Lataster used LSSRM (Alpert et al. 2003). SRTM implicitly assumes no time-dependent change in DA. The calculated endpoint, $\mathrm{BP}_{\mathrm{ND}}$, represents an average level of DA binding over the scan. Poor fitting of the data can lead to unreliable estimates of BP (see Sullivan et al. 2013). On the other hand, LSSRM allows for non-constant DA level during the scan. LSSRM models DA release as an exponential decay that peaks instantaneously at the start of the stimulus. The calculated endpoint, $\gamma$, represents the leading coefficient of the exponential, i.e. the peak DA level.

Figure 4 is a side-by-side comparison of the t-maps published in the two studies. The t-scores were derived from parameters $\mathrm{BP}_{\mathrm{ND}}$ and $\gamma$, estimated by SRTM and LSSRM, respectively. The t-scores represent effect sizes for DA release during the task, according to the two models. Despite similar experimental designs, the reader should note the considerable differences in the t-maps for the two studies. The effect sizes from the Lataster analysis are considerably greater than those of Nagano-Saito, as indicated both by the spatial extent of, and t-scores within the thresholded clusters. The differences in the strength of the findings lie in the differences in the capacities of the kinetic models to describe the data (Yoder et al. 2004). More robust detection of the effect by LSSRM over SRTM suggests that a time-varying model for DA release is the preferred descriptor of the data from a study using the MIST.

We acknowledge there are differences between the studies. Nagano et al. used a two-scan design in which control and stress scans were acquired on separate days. Lataster acquired control data and stress data within a single scan. There were slight differences in cohort demographics, data smoothing, and corrections for multiple comparisons. Despite these differences, it is most important to understand that the t-scores in Fig. 4 are uncorrected values. Thus, they can be compared directly to one another. Different methods of correcting for multiple comparisons may differentially affect the t-score threshold (determining significance) but the threshold would only affect the spatial extent of the clusters and not the intensity values. As can be seen in Fig. 4, the $\mathrm{t}$-scores from LSSRM analysis are considerably higher than SRTM. In fact, there is minimal overlap between the ranges of t-scores from the two studies.

LSSRM appears to be more sensitive to transient DA than SRTM, but it may not be ideal for describing the widest range of possible DA responses. DA responses with unknown take-off or peak times will not be well-modeled. The limitations of LSSRM derive from its stipulations that (1) DA peaks instantaneously and (2) the shape of the DA curve is a decaying exponential. A model which allows the DA curve to take on a variety of forms-namely, allowing

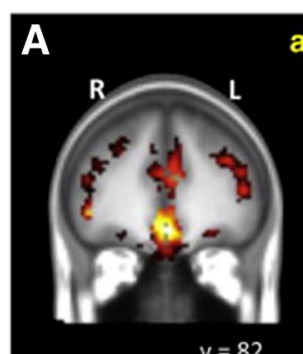

a
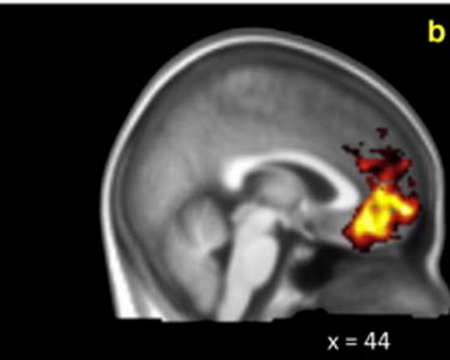

c
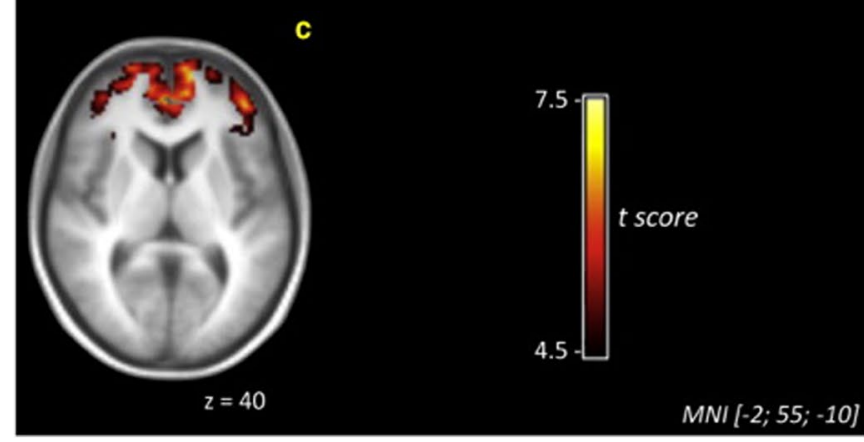

Fig. 4 DA release during MIST. (A) t-score map based on $\gamma$ as estimated with LSSRM from Lataster et al., (B) t-score map based on $\mathrm{BP}_{\mathrm{ND}}$ as estimated with SRTM from Nagano-Saito et al. Used with

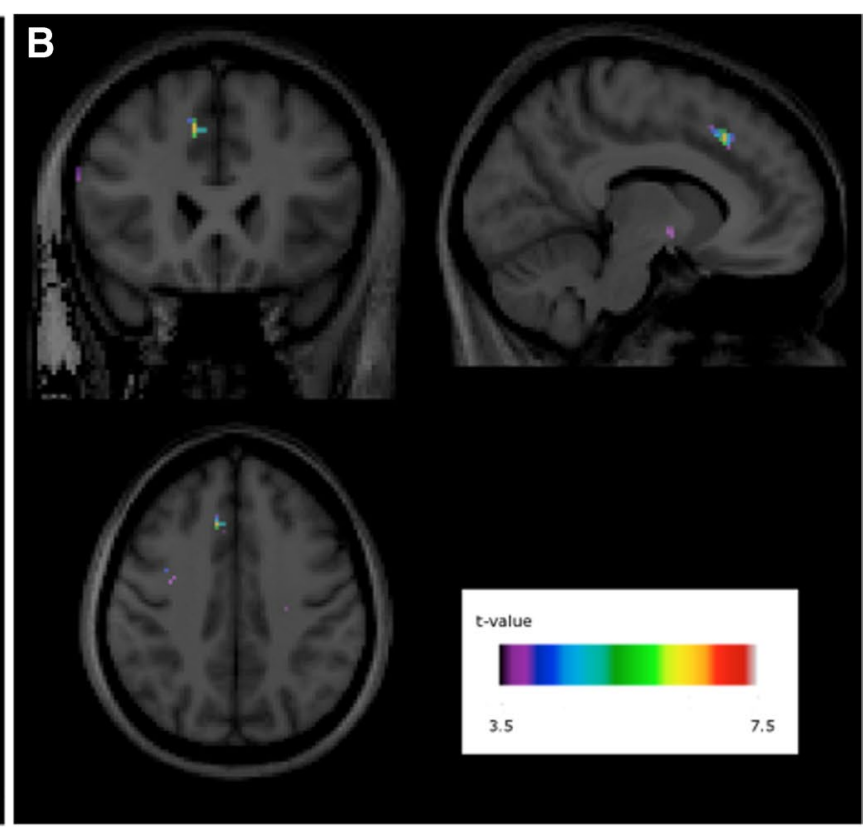

publisher's permission from Lataster et al. (2011), Neuroimage and Nagano-Saito et al. (2013), Synapse 
peak DA concentration to occur sometime after the start of the task-could be more widely applicable and potentially more robust. The lp-ntPET model (Eq. 1) fits this condition. In addition, lp-ntPET allows for varying response start times $\left(t_{D}\right)$, including negative $t_{D}$. A negative $t_{D}$ would indicate dopamine release starting before the start of the pharmacological stimulus. This might be the case if anticipation of reward were the dominant dopaminergic phenomenon. The application of lp-ntPET to cortical DA data is the next logical step to our goal of quantification of the spatiotemporal patterns of DA release in the entire corticolimbic circuit.

\section{Special considerations for creating whole-brain DA movies}

The application of kinetic models that are not optimally suited to the PET data may result in high variability in calculated endpoints and diminished sensitivity for detection of stimulus-induced DA release. The deficiency in conventional models for describing data acquired by Brody et al. with consistency also likely explains the more robust findings from Lataster et al. (2011) compared to NaganoSaito et al. The time-invariant model, SRTM, was unable to reliably capture the presumed time-varying DA component of the PET signal. On the other hand, the lp-ntPET model was able to characterize the spatiotemporal patterns of DA release during smoking with RAC PET Cosgrove, Wang et al. (2014). We are now preparing to extend the high sensitivity of lp-ntPET to create DA movies of the cortex - and ultimately the whole brain.

We anticipate challenges in the development of wholebrain DA movies. Simulations can be used in optimization processes as we did previously in the development of striatal DA movies (Wang et al. 2016). Microdialysis data of cortical DA (Narendran et al. 2014; Tanda et al. 2015; Jedema et al. 2014) can guide the creation of realistic simulations of extra-striatal DA responses. Simulations can also help to determine optimal binding kinetics for the tracer and optimal timing for the stimulus. Both are important for maximizing sensitivity (Wang et al. 2016). Full characterization of the corticostriatal DA response may require the use of RAC in the striatum and a high affinity DA tracer for the cortex. This, in turn, would require a means of synchronizing data from two scans sessions. Despite the challenges, we believe that 'whole-brain DA movies' will help us to visualize the entire corticolimbic circuitry and how its dysfunction might result in addiction.

Acknowledgements We thank Dr. Lihan Yan for her support and helpful discussions. We thank Dr. Paul Gravel for his support and help in assembling dopamine movies.

\section{Compliance with Ethical Standards}

Funding This work was supported by grants from the National Institutes of Health; Contract grant numbers: R21DA032791 (to EDM), 1R01DA038709 (to EDM), 1R21DA040852 (to EDM), and K02DA031750 (to KPC). HL was supported by the Biomedical Engineering Program at Yale University and Robert E. Apafel Fellowship. YZ was supported by the Interdepartmental Neuroscience Training Program at Yale University (T32NS4122813), Gruber Science Foundation Fellowship and the National Science Foundation Graduate Research Fellowship Program (NSF-GRFP).

Conflict of interest The authors declare that they have no conflict of interest.

Ethical approval and informed consent This is a review article and therefore does not contain any new data from studies with human participants or animals performed by any of the authors. To the best of the author's knowledge, the data reviewed in this article were all acquired in accordance with the ethical standards of the relevant institutional and/or national research committee and with the 1975 Helsinki declaration and its later amendments or comparable ethical standards.

Open Access This article is distributed under the terms of the Creative Commons Attribution 4.0 International License (http://creativecommons.org/licenses/by/4.0/), which permits unrestricted use, distribution, and reproduction in any medium, provided you give appropriate credit to the original author(s) and the source, provide a link to the Creative Commons license, and indicate if changes were made.

\section{References}

Alpert, N. M., Badgaiyan, R. D., Livni, E., \& Fischman, A. J. (2003). A novel method for noninvasive detection of neuromodulatory changes in specific neurotransmitter systems. NeuroImage, 19, 1049-1060.

Berridge, Kent C. (2007). The debate over dopamine's role in reward: the case for incentive salience. Psychopharmacology, 191, $391-431$.

Brody, A. L., Mandelkern, M. A., Olmstead, R. E., Scheibal, D., Hahn, E., Shiraga, S., Zamora-Paja, E., Farahi, J., Saxena, S., London, E. D., \& McCracken, J. T. (2006). Gene variants of brain dopamine pathways and smoking-induced dopamine release in the ventral caudate/nucleus accumbens. Archives of General Psychiatry, 63, 808-16.

Brody, Arthur L., Edythe, D., London, Richard E., Olmstead, Zoe, Allen-Martinez, Stephanie, Shulenberger, Matthew R., Costello, Anna L., Abrams, David Scheibal, Judah Farahi, Steven Shoptaw, \& Mandelkern M. A. (2010) Smoking-induced change in intrasynaptic dopamine concentration: effect of treatment for tobacco dependence. Psychiatry Research: Neuroimaging, 183, 218-24.

Brody, Arthur L., Mark, A., Mandelkern, Murray E., Jarvik, G. S., Lee, Erlyn C., Smith, J. C., Huang, R. G., Bota, George, \& Bartzokis, and London, E. D. (2004). Differences between smokers and nonsmokers in regional gray matter volumes and densities. Biological Psychiatry, 55, 77-84.

Carson, R. E., Barker, W. C., Liow, J. S., \& Johnson, C. A. (2004). Design of a motioncompensation OSEM list-mode algorithm for resolution-recovery reconstruction for the HRRT. IEEE Nuclear Science Symposium, 3281-3285. Portland, OR. 
Cosgrove, K. P., Wang, S., Kim, S. J., McGovern, E., Nabulsi, N., Gao, H., Labaree, D., Tagare, H. D., Sullivan, J. M. \& Morris, E. D. (2014). Sex differences in the brain's dopamine signature of cigarette smoking. The Journal of Neuroscience: The Official Journal of the Society for Neuroscience, 34, 16851-16855.

Dedovic, K., Renwick, R., Mahani, N. K., Engert, V., Lupien, S. J., \& Pruessner, J. C. (2005). The montreal imaging stress task: using functional imaging to investigate the effects of perceiving and processing psychosocial stress in the human brain. Journal of Psychiatry \& Neuroscience: JPN, 30, 319-25.

Dichter, Gabriel S., Cara, A., Damiano, \& Allen, John A. (2012). Reward circuitry dysfunction in psychiatric and neurodevelopmental disorders and genetic syndromes: animal models and clinical findings. Journal of Neurodevelopmental Disorders, 4, 19-19.

Egerton, A., Mehta, M. A., Montgomery, A. J., Lappin, J. M., Howes, O. D., Reeves, S. J., Cunningham, V. J. \& Grasby, P. M. (2009). The dopaminergic basis of human behaviors: a review of molecular imaging studies. Neuroscience and Biobehavioral Reviews, 33, 1109-1132.

Farde, L., Hall, H., Ehrin, E., \& Sedvall, G. (1986). Quantitative analysis of $\mathrm{D} 2$ dopamine receptor binding in the living human brain by PET. Science, 231, $258-61$.

Hall, H., Kohler, C., Gawell, L., Farde, L., \& Sedvall, G. (1988). Raclopride, a new selective ligand for the dopamine-D2 receptors. Progress in Neuro-Psychopharmacology \& Biological Psychiatry, 12, $559-68$.

Ichise, M., Liow, J. S., Lu, J. Q., Takano, A., Model, K., Toyama, H., Suhara, T., Suzuki, K., Innis, R. B., \& Carson, R. E. (2003). Linearized reference tissue parametric imaging methods: application to [11C]DASB positron emission tomography studies of the serotonin transporter in human brain. Journal of Cerebral Blood Flow and Metabolism: Official Journal of the International Society of Cerebral Blood Flow and Metabolism, 23, 1096 - 112.

Imperato, P. J., \& Mitchell, G. (1986). Cigarette smoking: a "chosen" risk. New York State Journal of Medicine, 86, 485-489.

Innis, R. B., Cunningham, V. J., Delforge, J., Fujita, M., Gjedde, A., Gunn, R. N., Holden, J., Houle, S., Huang, S. C., Ichise, M., Iida, H., Ito, H., Kimura, Y., Koeppe, R. A., Knudsen, G. M., Knuuti, J., Lammertsma, A. A., Laruelle, M., Logan, J., Maguire, R. P., Mintun, M. A., Morris, E. D., Parsey, R., Price, J. C., Slifstein, M., Sossi, V., Suhara, T., Votaw, J. R., \& Wong, D. F., Carson, R. E. (2007). Consensus nomenclature for in vivo imaging of reversibly binding radioligands. Journal Cerebral Blood Flow Metabolism, 27, 1533-1539.

Jedema, Hank P., Narendran, Rajesh, \& Bradberry, Charles W. (2014). Amphetamine-induced release of dopamine in primate prefrontal cortex and striatum: striking differences in magnitude and timecourse. Journal of Neurochemistry, 130, 490 - 97.

Kebabian, John W., Gary, L., Petzold, \& Greengard, Paul. (1972). Dopamine-sensitive adenylate cyclase in caudate nucleus of rat brain, and its similarity to the "dopamine receptor". Proceedings of the National Academy of Sciences of the United States of America, 69, 2145-49.

Kim, Su, Jin, Jenna M., Sullivan, Shuo, Wang, K. P., Cosgrove, \& Morris, Evan D. (2014). Voxelwise lp-ntPET for detecting localized, transient dopamine release of unknown timing: sensitivity analysis and application to cigarette smoking in the PET Scanner. Human Brain Mapping, 35, 4876-4891.

Koepp, M. J., Gunn, R. N., Lawrence, A. D., Cunningham, V. J., Dagher, A., Jones, T., Brooks, D. J., Bench, C. J. \& Grasby, P. M. (1998). Evidence for striatal dopamine release during a video game. Nature, 393, 266-268.

Lammertsma, A. A., \& Hume, S. P. (1996). Simplified reference tissue model for PET receptor studies. NeuroImage, 4, 153-158.

Lataster, Johan, Collip, Dina, Ceccarini, Jenny, Haas, David, Linda Booij, Jim van Os, Jens Pruessner, Koen Van Laere, \& Inez
Myin-Germeys. (2011) Psychosocial stress is associated with in vivo dopamine release in human ventromedial prefrontal cortex: a positron emission tomography study using [18F]fallypride. NeuroImage, 58, 1081-1089.

Morris, E. D., Normandin, M. D., \& Schiffer, W. K. (2008). Initial comparison of ntPET with microdialysis measurements of methamphetamine-induced dopamine release in rats: support for estimation of dopamine curves from PET data. Molecular Imaging and Biology: MIB: The Official Publication of the Academy of Molecular Imaging, 10, 67-73.

Morris, E. D., \& Yoder, K. K. (2007). Positron emission tomography displacement sensitivity: predicting binding potential change for positron emission tomography tracers based on their kinetic characteristics. Journal of Cerebral Blood Flow and Metabolism: Official Journal of the International Society of Cerebral Blood Flow and Metabolism, 27, 606-17.

Morris, E. D., Yoder, K. K., Wang, C., Normandin, M. D., Zheng, Q. H., Mock, B., Muzic, R. F. Jr., \& Froehlich, J. C. (2005). ntPET: a new application of PET imaging for characterizing the kinetics of endogenous neurotransmitter release. Molecular Imaging: Official Journal of the Society for Molecular Imaging, $4,473-89$.

Mukherjee, J., Yang, Z. Y., Brown, T., Lew, R., Wernick, M., Ouyang, X., Yasillo, N., Chen, C. T., Mintzer, R., \& Cooper, M. (1999). Preliminary assessment of extrastriatal dopamine D-2 receptor binding in the rodent and nonhuman primate brains using the high affinity radioligand, 18F-fallypride. Nuclear Medicine and Biology, 26, $519-27$.

Nagano-Saito, Atsuko, Dagher, Alain, Booij, Linda, Gravel, Paul, Welfeld, Krzysztof, Kevin, F., Casey, Marco Leyton, \& Chawki Benkelfat. (2013). Stress-induced dopamine release in human medial prefrontal cortex-18F-Fallypride/PET study in healthy volunteers, Synapse, 67, $821-30$.

Narendran, R., Jedema, H. P., Lopresti, B. J., Mason, N. S., Gurnsey, K., Ruszkiewicz, J., Chen, C. M., Deuitch, L., Frankle, W. G. \& Bradberry, C. W. (2014). Imaging dopamine transmission in the frontal cortex: a simultaneous microdialysis and [11C]FLB 457 PET study. Molecular Psychiatry, 19, 302 - 10.

Normandin, M. D., \& Morris, E. D. (2006). Temporal resolution of ntPET using either arterial or reference region-derived plasma input functions. Conference Proceedings: Annual International Conference of the IEEE Engineering in Medicine and Biology Society. IEEE Engineering in Medicine and Biology Society. Conference, 1, 2005-2008.

Normandin, M. D., Schiffer, W. K., \& Morris, E. D. (2012). A linear model for estimation of neurotransmitter response profiles from dynamic PET data, NeuroImage, 59, 2689-2699.

Olsson, H., Halldin, C., Swahn, C. G., \& Farde, L. (1999). 'Quantification of [11C]FLB 457 binding to extrastriatal dopamine receptors in the human brain'. Journal of Cerebral Blood Flow and Metabolism: Official Journal of the International Society of Cerebral Blood Flow and Metabolism, 19, 1164-1173.

Perkins, K. A., Grobe, J. E., Weiss, D., Fonte, C., \& Caggiula, A. (1996). Nicotine preference in smokers as a function of smoking abstinence. Pharmacology, Biochemistry, and Behavior, 55, $257-63$.

Perkins, Kenneth A., Jacobs, Lynette, Sanders, Mark, \& Caggiula, Anthony R. (2002). Sex differences in the subjective and reinforcing effects of cigarette nicotine dose. Psychopharmacology, 163, 194-201.

Robinson, Terry E., \& Berridge, Kent C. (1993). The neural basis of drug craving: An incentive-sensitization theory of addiction. Brain Research Reviews, 18, 247 - 91.

Rollema, Hans, Coe, Jotham W., Leslie, K., Chambers, R. S., Hurst, Stephen M., Stahl, \& Williams, Kathryn E. (2007). Rationale, pharmacology and clinical efficacy of partial agonists of $\alpha 4 \beta 2$ 
nACh receptors for smoking cessation. Trends in Pharmacological Sciences, 28, $316-25$.

Salamone, John D., \& Mercè, Correa (2002). Motivational views of reinforcement: implications for understanding the behavioral functions of nucleus accumbens dopamine. Behavioural Brain Research, 137, 3-25.

Schultz, Wolfram. (2007). Multiple dopamine functions at different time courses. Annual Review of Neuroscience, 30, 259-88.

Sullivan, Jenna M., Kim, Su Jin, Kelly, P., Cosgrove, \& Morris, Evan D. 2013. 'Limitations of SRTM, Logan graphical method, and equilibrium analysis for measuring transient dopamine release with [(11)C]raclopride PET'., American Journal of Nuclear Medicine and Molecular Imaging, 3, 247 - 60.

Swanson, L. W. (1982). The projections of the ventral tegmental area and adjacent regions: a combined fluorescent retrograde tracer and immunofluorescence study in the rat. Brain Research Bulletin, 9, $321-53$.

Tanda, G., Valentini, V., De Luca, M. A., Perra, V., Serra, G. P., \& Di Chiara, G. (2015). A systematic microdialysis study of dopamine transmission in the accumbens shell/core and prefrontal cortex after acute antipsychotics. Psychopharmacology (Berl), 232, $1427-1440$.
Volkow, Nora D., \& Swanson, James M. (2003). Variables that affect the clinical use and abuse of methylphenidate in the treatment of ADHD'. American Journal of Psychiatry, 160, 1909-1918.

Wang, S., Kim, S., Cosgrove, K. P., \& Morris, E. D. (2016). A framework for designing dynamic lp-ntPET studies to maximize the sensitivity to transient neurotransmitter responses to drugs: Application to dopamine and smoking', Neuroimage.

Watabe, H., Endres, C. J., Breier, A., Schmall, B., Eckelman, W. C., $\&$ Carson, R. E. (2000). Measurement of dopamine release with continuous infusion of [11C]raclopride: optimization and signalto-noise considerations. Journal of Nuclear Medicine: Official Publication, Society of Nuclear Medicine, 41, 522 - 30.

Willuhn, Ingo, Wanat, Matthew J., Jeremy, J., Clark, \& Paul, E. M., $\&$ Phillips (2010). Dopamine signaling in the nucleus accumbens of animals self-administering drugs of abuse.'. In David W. Self, Julie K. Staley \& Gottschalk (Eds.), Behavioral Neuroscience of Drug Addiction. Berlin Heidelberg, Springer.

Yoder, K. K., Wang, C., \& Morris, E. D. (2004). Change in binding potential as a quantitative index of neurotransmitter release is highly sensitive to relative timing and kinetics of the tracer and the endogenous ligand. Journal of Nuclear Medicine: Official Publication, Society of Nuclear Medicine, 45, 903 - 11. 